\title{
CATEGORIES OF $V$-SETS
}

\author{
BY J. A. GOGUEN ${ }^{1}$
}

Communicated by Saunders Mac Lane, December 9, 1968

Let $V$ be a partially ordered set. Then a $V$-set is a function $A: X$ $\rightarrow V$ from a set $X$ to $V$. $V$ is the set of values for $A$, and $X$ is the carrier of $A$. If $B: Y \rightarrow V$ is another $V$-set, a morphism $f: A \rightarrow B$ is a function $\bar{f}: X \rightarrow Y$ such that $A(x) \leqq B(\bar{f}(x))$ for each $x \in X$. The category of all $V$-sets is denoted $\delta(V)$. The carrier functor $K: \delta(V) \rightarrow S$ assigns $X$ to $A: X \rightarrow V$ and $\bar{f}: X \rightarrow Y$ to $f: A \rightarrow B$, where $S$ is the category of sets. See [2].

If $V$ has one point, $\delta(V)=\delta$. If $V=\{0,1\}$, where $0<1, \delta(V)$ is the category of pairs $(X, A)$ of sets, where $A \subseteq X$. If $V$ is the closed unit interval, $s(V)$ is the category of "fuzzy sets", as used by Zadeh and others [1], [5] for problems of pattern recognition and systems theory. When $V$ is a Boolean algebra, $V$-sets are Boolean-valued sets, as used by Scott and Solovay for independence results in set theory (however, their notion of morphism is different).

If $V$ is complete, $\mathfrak{s}(V)$ is a pleasant category satisfying all Lawvere's axioms [3] for $S$ except choice, modulo some substitutions of the $V$-set with carrier 1 and value 0 for the terminal object. In particular,

Theorem 1. If $V$ is complete, $s(V)$ is complete and cocomplete, has an exponential (i.e., a coadjoint to product) and a "Dedekind-Pierce object" (i.e., an object which looks like the set of integers; see [3]).

Let Poc denote the category of partially ordered classes, and let $\mathscr{L}$ be a subcategory of Poc. Then a category $\mathfrak{C}$ is $\mathscr{L}$-ordered if the power function $\rho:|\mathfrak{e}| \rightarrow$ Poc factors through $\&$, where $\mathcal{P}(A)$ is the class of all equivalence classes of monics with codomain $A(f \equiv g$ if $\exists$ an isomorphism $h$ such that $f h=g$ ). Denote the image of $A \stackrel{f}{\rightarrow} B$ by $f(A)$, and the image of the composite $A^{\prime} \stackrel{i}{\rightarrow} A \stackrel{f}{\rightarrow} B$, where $i$ is monic, by $f\left(A^{\prime}\right)$. Then $\mathcal{C}$ has associative images if it has images such that $f(g(A))$ $=(f g)(A)$, whenever $A \stackrel{g}{\rightarrow} B \stackrel{f}{\rightarrow} C$. $P$ can be construed as a functor when $\mathcal{C}$ has associative images. Let $\mathrm{CL}$ denote the category of complete lattices, and call a category $C_{1}$ if a coproduct of monics is always monic.

\footnotetext{
${ }^{1}$ Research supported by Office of Naval Research under contracts Nos. 3656(08)
} and 222(85), at the University of California at Berkeley. 
THEOREM 2. A CL-ordered category with associative images has equalizers, inverse images, unions, intersections, and epic images. If it has coproducts, it is $C_{1}$.

An object $P$ in a category $\mathcal{C}$ is monic if every arrow $P \rightarrow A$ is monic, and is further atomic if every $P \rightarrow A$ is atomic in $P(A) . P$ is good if the functor $[P]:, \mathbb{C} \rightarrow \mathcal{S}$ is noninitial preserving. A union $U_{i} A_{i}$ in $\mathrm{C}$ is disjoint if $i \neq j \Rightarrow A_{i} \cap A_{j}=\varnothing$, where $\varnothing$ is the initial object. Let CDL be the category of completely distributive lattices, i.e., complete lattices satisfying the law $a \wedge V_{i} b_{i}=V_{i}\left(a \wedge b_{i}\right)$. Such lattices $V$ have pseudo-complement operators ${ }^{*}: V \rightarrow V$ defined by $a^{*}=\mathrm{V}\{b \mid a \wedge b=0\}$. Call $V \in|C D L|$ disjointed if for each pair $x, y$ of unequal atoms, $x^{*} \vee y^{*}=I$, the maximal element of $V$, and call $\mathrm{e}$ disjointedly $C D L$ ordered if each $\odot(A) \in|C D L|$ is disjointed.

THEOREM 3. A category $\mathfrak{e}$ is equivalent to $\mathrm{S}(V)$ for some $V \in|C D L|$ if and only if:

(1) $\mathfrak{C}$ has an atomic monic good projective generator $P$;

(2) e has initial and terminal objects, $\varnothing$ and $I$, respectively;

(3) e has coproducts, which are disjoint unions; and conversely, each disjoint union in $\mathbb{C}$ is a coproduct in $\mathfrak{C}$;

(4) e has associative images;

(5) $\mathcal{e}$ is disjointedly CDL-ordered; and

(6) $P \Perp P$ is not isomorphic to $P$.

The Axioms (1)-(6) are easily verified for $s(V), V \in|C D L|$. We now sketch the converse, which (surprisingly) makes no use of adjoint functors. Essential use is made of Theorem 2, via Axioms (4) and (5).

Call the elements of $[P, A]$ the points of $A$. We first show the onepointed objects of $\mathcal{C}$ are the subobjects of $I$, except $\varnothing$; denote this lattice $V$. A calculation shows that each $A \in|\mathbb{e}|$ is a disjoint union $\bigcup_{x \in[P, A]^{x_{* *}}}$, so by Axiom (3), $A=\bar{\amalg}_{x \in[P, A]^{x_{* *}} \text {. These facts combine }}$ to show that each $A$ is a subobject of $I^{[P, A]}$, the coproduct of $I$ over the index set $[P, A]$. We then show the arrows $f: A \rightarrow B$ in $\mathcal{C}$ are in 1-1 correspondence with appropriate arrows $\bar{f}:[P, A] \rightarrow[P, B]$ in $S$. The functor $E: \mathfrak{e} \rightarrow S(V)$ defined by $K(E(A))=[P, A], E(A)(x)=x^{* *} \in V$, and $E(f)=[P, f]$, is then shown to be full, faithful, and representative.

The addition to Axioms (1)-(6) of either the categorical axiom of choice, or the condition $I=P$, yields a characterization of $s$. For finite distributive lattices $V$, categories of $V$-sets with finite carrier are similarly characterized by all elementary axioms. 


\section{REFERENCES}

1. R. Bellman, R. Kalaba and L. A. Zadeh, Abstraction and pattern classification, J. Math. Anal. Appl. 13 (1966), 1-7.

2. J. A. Goguen, L-fuzzy sets, J. Math. Anal. Appl. 18 (1967), 145-174.

3. F. W. Lawvere, An elementary theory of the category of sets, Proc. Nat. Acad. Sci. U.S.A. 52 (1964), 1506-1511.

4. D. Scott and R. Solovay, Boolean valued models for set theory (to appear).

5. L. A. Zadeh, Fuzzy sets, Information and Control 8 (1965), 338-353.

University of Chicago, Chicago, Illinois 60637

\section{ERRATUM}

\section{Volume 75}

Y.-T. Siu, Extending coherent analytic sheaves through subvarieties, pp. 123-126.

\section{Page 125:}

Line 3. $\tilde{\theta}^{p} / \tilde{f}^{p}$ should read $\tilde{\theta}^{p} / f \tilde{\theta}^{p}$.

Line 9. $\alpha\left(t_{m}^{i}\right)=s_{m}^{i}$ should read $\alpha\left(t_{m}^{i}\right)=s_{m}^{i}-\sum_{q=1}^{m-1} a_{q} s_{a}^{(t)}$.

Line 10 from bottom. $\mathbf{C}^{N}$ should read $\mathbf{C}^{n}$. 